\title{
Parabiosis reveals leukocyte dynamics in the kidney
}

\author{
Jeremie M Lever ${ }^{1,2}$, Zhengqin Yang ${ }^{1,2}$, Ravindra Boddu ${ }^{1,2}$, Oreoluwa O Adedoyin 1,2, Lingling Guo ${ }^{1,2}$, Reny Joseph ${ }^{1,2}$, \\ Amie M Traylor ${ }^{1,2}$, Anupam Agarwal ${ }^{1,2,3}$ and James F George 2,4
}

The immune cellular compartment of the kidney is involved in organ development and homeostasis, as well as in many pathological conditions. Little is known about the mechanisms that drive intrarenal immune responses in the presence of renal tubular and interstitial cell death. However, it is known that tissue-resident leukocytes have the potential to have distinct roles compared with circulating cells. We used a parabiosis model in C57BL/6 CD45 congenic and green fluorescent protein transgenic mice to better understand the dynamics of immune cells in the kidney. We found $\mathrm{F} 4 / 80^{\mathrm{Hi}}$ intrarenal macrophages exhibit minimal exchange with the peripheral circulation in two models of parabiosis, whether mice were attached for 4 or 16 weeks. Other intrarenal inflammatory cells demonstrate near total exchange with the circulating immune cell pool in healthy kidneys, indicating that innate and adaptive immune cells extensively traffic through the kidney interstitium during normal physiology. Neutrophils, dendritic cells, F4/80 Low macrophages, T cells, B cells, and NK cells are renewed from the circulating immune cell pool. However, a fraction of double-negative T (CD4 CD8 ${ }^{-}$) and NKT cells are long-lived or tissue resident. This study provides direct evidence of leukocyte sub-populations that are resident in the renal tissue, cells which demonstrate minimal to no exchange with the peripheral blood. In addition, the data demonstrate continual exchange of other sub-populations through uninflamed tissue.

Laboratory Investigation (2018) 98, 391-402; doi:10.1038/labinvest.2017.130; published online 18 December 2017

Inflammatory cells have an important role in many kidney diseases, including acute kidney injury (AKI), glomerulonephritis, acute interstitial nephritis, tubulointerstitial fibrosis, lupus nephritis, diabetic nephropathy, and hypertensive kidney disease. ${ }^{1-5}$ Leukocytes are present in healthy kidneys and their numbers increase in inflamed and diseased kidneys. $^{6-10}$ Observable cell types include neutrophils (PMN), various subtypes of macrophages and dendritic cells (DCs), T cells, B cells, NK cells, and NKT cells, among others. Following injurious events, immune cells are responsible for coordinating tissue regeneration, ${ }^{6}$ or alternatively, propagating tissue damage and increasing propensity toward eventual fibrosis and scarring. ${ }^{11,12}$

Several laboratories have studied the phenotype, gene expression profiles, and functional role of mononuclear phagocytes (MP) in kidney disease. ${ }^{6,8,10,13-17}$ MP include monocytes, macrophages, and DCs. Studies of kidney MP suggest the existence of a tissue resident population, somewhat interchangeably called macrophages or DC, which may have protective or pro-resolving roles. ${ }^{6,10,13,14,16,18}$ No consensus exists as to which MP sub-populations are resident and which exchange with the peripheral blood. Embryogenesis studies show that intrarenal $\mathrm{F} 4 / 80^{+}$macrophages arise during development first from the embryonic yolk sac and later from the fetal liver. ${ }^{8,19-21}$ Similar intrarenal MP persist into adulthood and appear to undergo in situ proliferation. , $^{82}$ Other renal MP subtypes arise from Myb-dependent bone marrow precursors that migrate into the kidney. ${ }^{19}$ Certain lymphoid sub-populations are also found in the healthy kidney, including NK cells ${ }^{23}$ and double-negative T lymphocytes (DNTL, CD3 $\left.{ }^{+} \mathrm{CD}^{-} \mathrm{CD}^{-}\right),{ }^{24}$ which appear to have varying functional roles in AKI.

To test the hypothesis that certain renal leukocytes, including $\mathrm{F} 4 / 80^{\mathrm{Hi}} \mathrm{MP}$, demonstrate little to no exchange with the peripheral blood, we applied the parabiosis model between two animals bearing different allotypic or fluorescent markers. ${ }^{25}$ This experimental system allows observation of exchange of immune cells between the peripheral blood and the tissues. We therefore applied it to formally establish within the limits of our experimental time frames, in adult

\footnotetext{
${ }^{1}$ Department of Medicine, University of Alabama at Birmingham, Birmingham, AL, USA; ${ }^{2}$ Nephrology Research and Training Center, University of Alabama at Birmingham, Birmingham, AL, USA; ${ }^{3}$ Department of Veterans Affairs, Birmingham, AL, USA and ${ }^{4}$ Department of Surgery, University of Alabama at Birmingham, Birmingham, AL, USA Correspondence: Dr A Agarwal, MD, Division of Nephrology, Department of Medicine, Room 647 THT, 1720 2nd Avenue South, University of Alabama at Birmingham, Birmingham, AL 35294

E-mail: agarwal@uab.edu or Dr JF George, PhD, Division of Cardiothoracic Surgery, Department of Surgery, Room 725 ZRB, 1720 2nd Avenue South, University of Alabama at Birmingham, Birmingham, Alabama 35294, US.

E-mail: jgeorge@uab.edu

Received 17 June 2017; revised 10 September 2017; accepted 21 September 2017
} 
mice, renal leukocyte sub-populations that are tissue resident or long-lived as opposed to those that traffic through the interstitium. Here we describe specific MP and lymphocyte sub-populations with diminished or nearly absent exchange with the peripheral blood. These tissue resident leukocytes are a promising target for therapy.

\section{MATERIALS AND METHODS Study Approval}

All procedures involving mice were performed in accordance with National Institutes of Health guidelines regarding the care and use of live animals and were reviewed and approved by the Institutional Animal Care and Use Committee of UAB.

\section{Animals}

Enhanced green fluorescent protein (GFP) mice were bred inhouse on a C57BL/6 background. C57BL/6J and B6.SJL-Ptprc ${ }^{a}$ $P e p c^{b} /$ BoyJ (CD45.1) mice were obtained from Jackson Laboratories. Mice matched for sex, age ( \pm 7 days), and body mass $( \pm 3 \mathrm{~g})$ were co-housed from the age of $4-5$ weeks, with a density no $>5$ mice per cage. The animals were monitored daily for evidence of fighting and were exposed to gel food diet supplement at least two times, 2-6 weeks before performance of parabiotic attachment. Animals were randomly assigned a pair member, with the appropriate transgene or CD45 congenic allotype, within their cohoused cohort. Pairs established for parabiotic attachment were "cage paired," meaning they were housed only with their future parabiotic pair member, 2 weeks before the parabiosis procedure, and the mice were observed again for aggressive behavior. Aggressive and injured mice were removed from the study. Using this husbandry protocol, aggression was highly uncommon, occurring in only 1 pair out of the 21 included in this study.

\section{Parabiosis Surgery}

Our protocol was adapted from that previously published by Kamran and colleagues. ${ }^{26}$ Pairs of mice were anesthetized with inhaled isoflurane, $1.5-2.0 \% \mathrm{v} / \mathrm{v}$ induction, $1.0-1.5 \% \mathrm{v} / \mathrm{v}$ maintenance. Fur was removed from the surgical site, which involved the entire flank region to be attached, from rostral of the cervical spine to rostral of the hip joint, as well as the involved extremities distally to the knee and elbow joints. Fur removal extended from $5 \mathrm{~mm}$ from the spinal column dorsally to $5 \mathrm{~mm}$ from the midline ventrally. Clippers were used to remove fur from the body, whereas hair removal cream was preferred for removal from extremities. The mice were laid supine and the site was disinfected with betadine followed by $70 \% \mathrm{EtOH}$. Incisions were made through the skin and muscular layer starting from the elbow joint and extended down the flank to the knee joint. Non-absorbable 3-0 interrupted sutures were placed around the knee and elbow joints for stabilization, with care taken to not obstruct blood flow to the distal extremities. Starting rostrally, interrupted sutures (4-7 sutures, or until very stable) were placed to attach the skin and muscular layers of the two parabionts in the area around the upper extremities, beginning and stabilizing the skin anastomosis. Working on the ventral side, continuous 5-0 absorbable vicryl sutures were placed through the skin and muscular layer, followed by the same through the dorsal skin and muscular layer. Buprenorphine analgesic was administered $(0.1 \mathrm{mg} / \mathrm{kg})$ s.c. immediately preoperatively and $12 \mathrm{~h}$ postoperatively. No oral antibiotics were used; however, topical antibiotic was applied to the surgical site upon completion of the procedure. Animals were provided with water in a plastic dish on the floor of the cage as well as moistened chow and gel food diet supplement (Bio-Serv Nutra-Gel Diet, $6.7 \mathrm{~g}$ per pair, 1/6 of $40 \mathrm{~g}$ cube) daily for 4 days after the procedure. During this period, body weight was recorded and mice observed for signs of delayed recovery, pain, distress, open wounds, or infection. From 5 days postprocedure, gel food diet was provided every other day, $6.7 \mathrm{~g}$ per pair, until 28 days after parabiosis.

\section{Flow Cytometry}

Leukocytes were isolated as previously described with minor modifications. $^{27}$ Mice were anesthetized with isoflurane $(2.5 \% \mathrm{v} / \mathrm{v}$ for induction, $1.5 \% \mathrm{v} / \mathrm{v}$ for maintenance) and perfused with $60 \mathrm{ml}$ of ice-cold phosphate buffered saline through the left ventricle after median thoracotomy to clear the kidney of leukocytes in the circulation. The kidneys were explanted, washed with PBS, and the capsule was removed. Each kidney was then weighed individually. Kidneys were minced into small pieces and enzymatically digested using 1.67 Wünsch U/ml Liberase DL (Roche Diagnostics, Indianapolis, IN, USA) in DMEM medium for $30 \mathrm{~min}$ at $37^{\circ} \mathrm{C}$. Cold PEB buffer (PBS, 2 mM EDTA, 1\% BSA) was added to disrupt calcium-dependent cell-cell complexes and to inactivate the Liberase DL enzyme (Roche). Disaggregation was completed by aspirating and expelling the suspension

\footnotetext{
Figure 1 Validation of the parabiosis model for study of renal inflammation. (a) Schematic representing animal husbandry and age at which mice undergo the parabiosis procedure. Twenty-eight-day parabiosis period is shown. (b) Development of CD45 parabiosis chimeras over time. Percent chimerism among blood leukocytes before parabiosis and after 14 and 28 days of parabiosis. Mean \pm s.e.m., $n=6$ pairs (six mice per group). (c) Gating strategy employed for identifying host and non-host chimeric viable leukocytes in the kidney of CD45 parabiosis chimeras. Circled kidneys represent sample used to generate histograms shown to the right. (d) Representative flow cytometry histograms for resolving host and non-host chimeric leukocytes in the kidney spleen and blood. Percent gated is shown for each region. (e) Photomicrograph of periodic acid-Schiff stained transverse section of 28-day parabiont kidney. Scale bars $1 \mathrm{~mm}$ (left panel) and $20 \mu \mathrm{m}$ (right panel). (f) Serum creatinine from unattached controls or CD45 parabionts after 28 days of parabiosis. Mean \pm s.e.m., $n=4-7$ for unattached controls, $n=16$ pairs for 28 days parabiosis.
} 
through $18 \mathrm{~g}$ then $20 \mathrm{~g}$ syringes in sequence. The suspension was passed through a $40-\mu \mathrm{m}$ nylon filter (Fisher) to remove undigested and fibrogenous material and then centrifuged at
$300 \mathrm{~g}$. Red blood cells were lysed using ACK lysis buffer at room temperature for $2 \mathrm{~min}$ for kidney, $3 \mathrm{~min}$ for spleen, and 5 min for blood, and then washed once with PBS. a

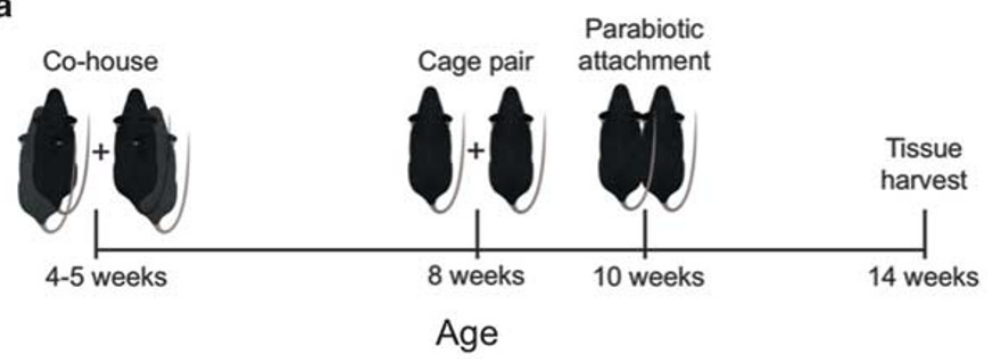

b

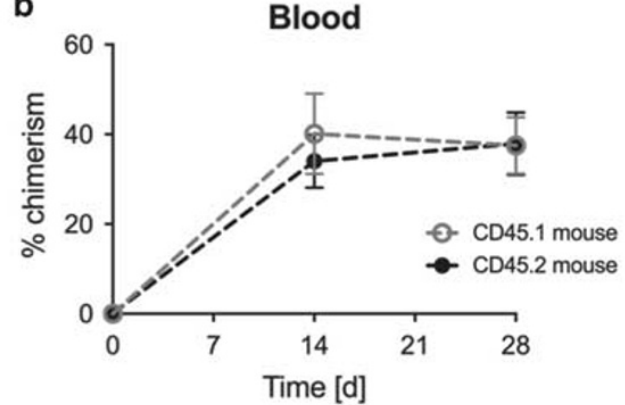

C
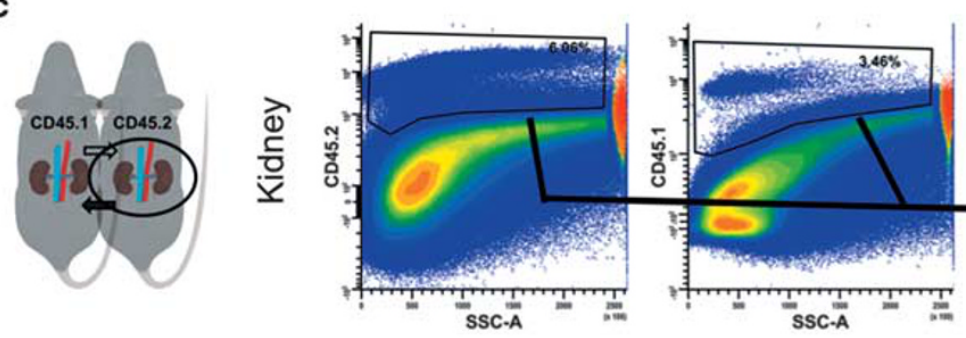

d

CD45 congenic

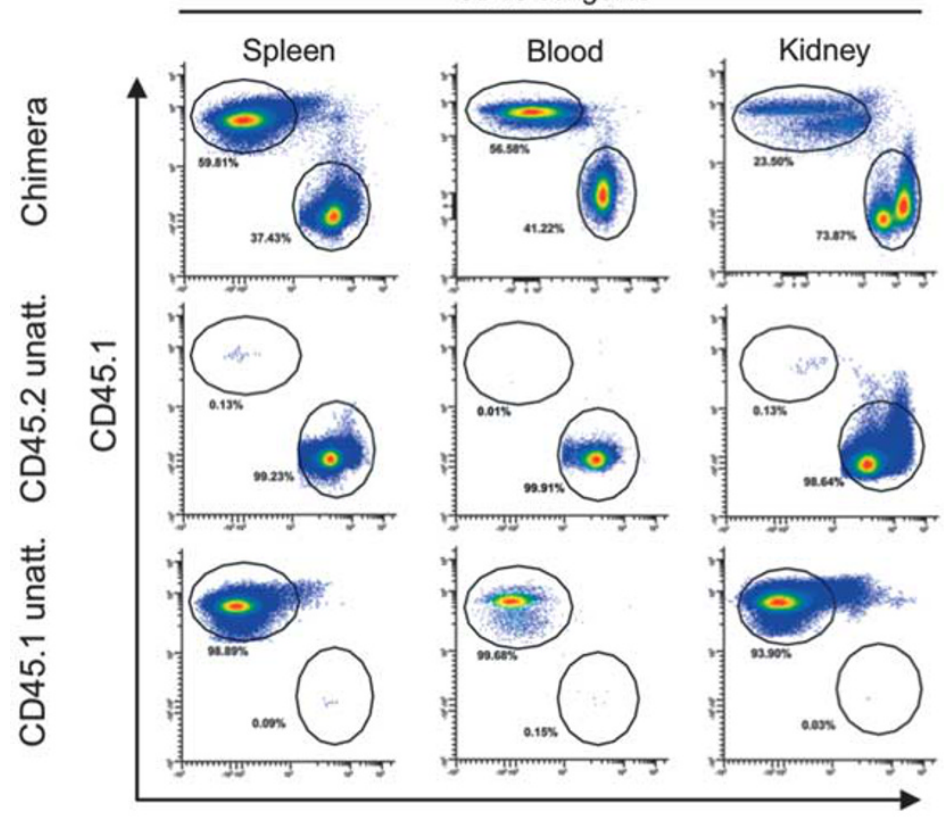

e

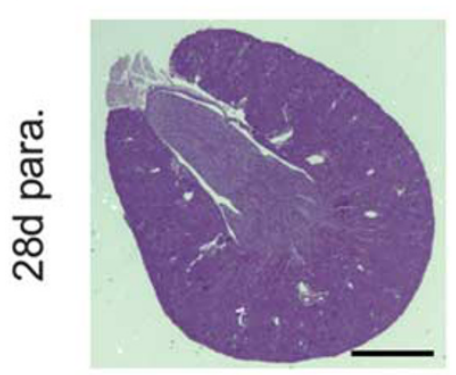

CD45.2
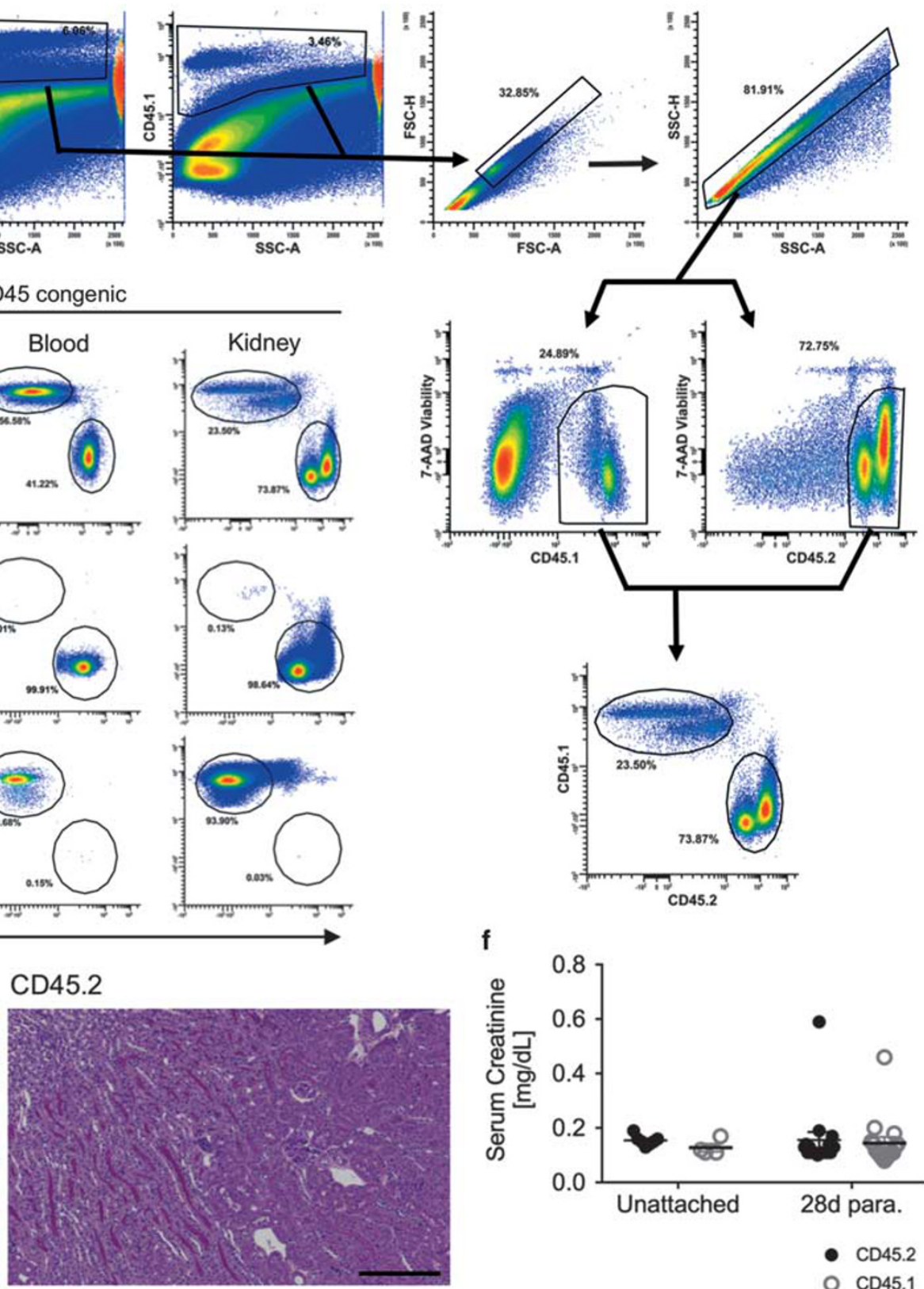

f

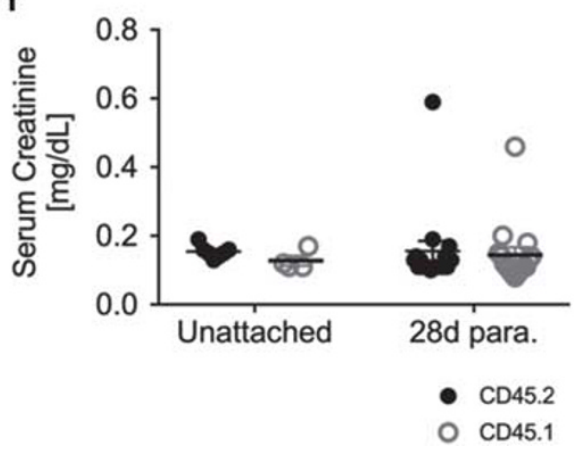


Leukocytes were stained according to the following protocol. Cells were washed with staining buffer (PBS, 0.5\% w/v BSA, 0.01\% w/v Sodium Azide) and incubated with antimouse CD16/32 (clone 93) for $10 \mathrm{~min}$ on ice to block nonspecific binding of $\mathrm{PE}$ to $\mathrm{Fc} 33$ receptors. Cells were stained with four independent panels for myeloid and lymphoid lineages in eGFP and CD45 parabionts. Myeloid eGFP parabiont cells were stained with PE-conjugated antiCD64a + b (Fcylr, clone NOD/Lt), 7-aminoactinomycin (7AAD), APC-conjugated anti-Gr-1 (Ly6G, clone 1A8), eFluor450-conjugated anti-Ly6C (HK1.4), eVolve605-conjugated anti-CD11b (M1/70), Brilliant Violet650-conjugated anti-CD45.2, and anti-CD45.1 (104, A20), APC-eFluor780-conjugated anti-F4/80 (BM8), Brilliant Violet785 anti-CD11c (N418). Lymphoid eGFP parabiont cells were stained with PEconjugated anti-NK1.1 (PK136), 7-AAD, APC-conjugated anti-CD3e (145-2C11), eFluor450-conjugated anti-CD8a (53-6.7), eVolve605-conjugated anti-CD4 (RM4-5), Brilliant Violet650-conjugated anti-CD45.2 and anti-CD45.1 (104, A20), APC-eFluor780-conjugated anti-MHCII (I-A/I-E, clone M5/114.15.2), and Brilliant Violet785-conjugated anti-CD19 (6D5). Myeloid CD45 parabiont cells were stained with FITCconjugated anti-CD45.1 (A20), PE-conjugated anti-CD64a + b (Fcylr, clone NOD/Lt), 7-AAD, APC-conjugated anti-Gr-1 (Ly6G, clone 1A8), eFluor450-conjugated anti-Ly6C (HK1.4), eVolve605-conjugated anti-CD11b (M1/70), Brilliant Violet650-conjugated anti-CD45.2 (104), APC-eFluor780conjugated anti-F4/80 (BM8), and Brilliant Violet785 antiCD11c (N418). Lymphoid CD45 parabiont cells were stained with FITC-conjugated anti-CD45.1 (A20), PE-conjugated anti-NK1.1 (PK136), 7-AAD, APC-conjugated anti-CD3e (145-2C11), eFluor450-conjugated anti-CD8a (53-6.7), eVolve605-conjugated anti-CD4 (RM4-5), Brilliant Violet650-conjugated anti-CD45.2 (104), APC-eFluor780conjugated anti-MHCII (I-A/I-E, clone M5/114.15.2), and Brilliant Violet785-conjugated anti-CD19 (6D5). Conjugated antibodies were purchased from EBioscience, Biolegend, and BD Biosciences.

Data were collected on a Becton-Dickenson LSR II at the UAB Comprehensive Flow Cytometry Core. Mixed fluorescence minus one and isotype controls of either kidney or splenic leukocytes were used to set positive gates. Results were analyzed using WinList Software (Version 7.0 and 8.0, Verity Software House Inc., Topsham, ME, USA).

\section{Microscopy}

Periodic acid-Schiff-hematoxylin-stained kidney sections were imaged using either a Nikon Eclipse TE2000-S or a Nikon SMZ800 microscope. Images were acquired and background-corrected using Nikon NIS Elements software (Version 4.60) and all images were color-adjusted in an identical manner in open source ImageJ software (Version 2.0.0).

\section{Kidney Function Measurements}

Serum creatinine was quantified using liquid chromatography tandem mass spectrometry (LC-MS/MS) as previously described. $^{28}$

\section{Statistical Analyses}

Statistical analyses were performed using Graphpad Prism software (Version 7.0, GraphPad Software, Inc., La Jolla, CA). Specific statistical tests applied were included in figure captions. Values of $P<0.05$ were considered statistically significant.

\section{RESULTS}

\section{Establishment of the Parabiosis Model}

In the CD45 congenic system, a C57BL/6J (CD45.2) mouse was surgically joined with an age and sex-matched CD45.1 congenic C57BL/6 mouse (Figure 1a). Following establishment of parabiosis, neovascularization occurs resulting in a shared peripheral circulation. We found after 14 days of parabiosis that $37.1 \pm 7.9 \%$ blood leukocytes in the paired mice were non-host, chimeric cells (values averaged from all parabionts), a value that was stable up to 4 weeks after attachment (Figure 1b), and chimeric leukocytes were observable in renal tissue but not unattached controls (Figures $1 \mathrm{c}$ and $\mathrm{d}$ ). Importantly, the state of parabiosis did not result in detectable changes in kidney structure (Figure 1e) or function (Figure 1f) 28 days after surgery.

In an alternative parabiosis model, we utilized transgenic mice on a C57BL/6 background that globally express GFP to validate our findings in the CD45 congenic system. ${ }^{29}$ One member of each pair was eGFP ${ }^{+/-}$and the other an $\mathrm{eGFP}^{-/-}$ littermate (Supplementary Figure 1a). In the eGFP system, we defined non-host, chimeric cells as eGFP ${ }^{+}$cells in the eGFP ${ }^{-\prime}$ - mouse, and conversely eGFP ${ }^{-}$cells in the $\mathrm{eGFP}^{+/-}$mouse. Chimeric $\mathrm{eGFP}^{+}$cells were detected in spleen, blood, and kidney after 4 weeks of parabiosis (Supplementary Figure 1b).

\section{Parabiosis Does Not Alter Leukocyte Proportions in Kidney or Spleen after 4 Weeks}

Validation of the parabiosis model as a tool for studying renal inflammation required that parabiosis does not cause systemic or renal inflammation. We measured immune cell proportions $\left(\% \mathrm{CD}^{+} 5^{+}\right)$in kidney and spleen, comparing parabionts to each other and to unattached control mice of identical genetic background. After 4 weeks of parabiosis, leukocyte proportions in CD45 and eGFP parabionts did not change relative to unattached controls (Figure 2a). Kidney macrophages made up the greatest proportion of intrarenal leukocytes (Figure 2a).

To determine if inflammation develops over time in the parabiotic mice, we measured leukocyte proportions after 16 weeks of parabiosis (Figure 2a) and compared them with values obtained for the same leukocyte populations at 4 weeks. In the kidneys of CD45 parabionts, the proportion of several cell types changed in comparison with 4-week parabionts, including $\mathrm{F} 4 / 80^{\mathrm{Hi}}$ macrophages, $\mathrm{B}$ cells, CD4s, 
a

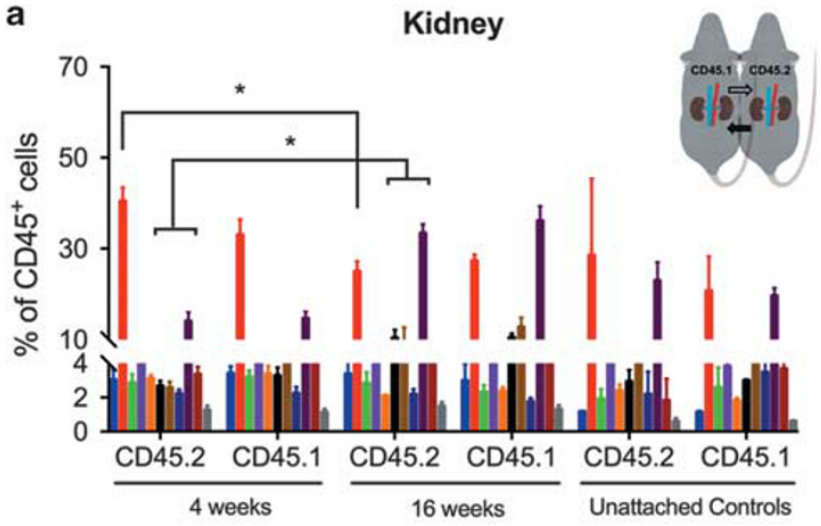

b

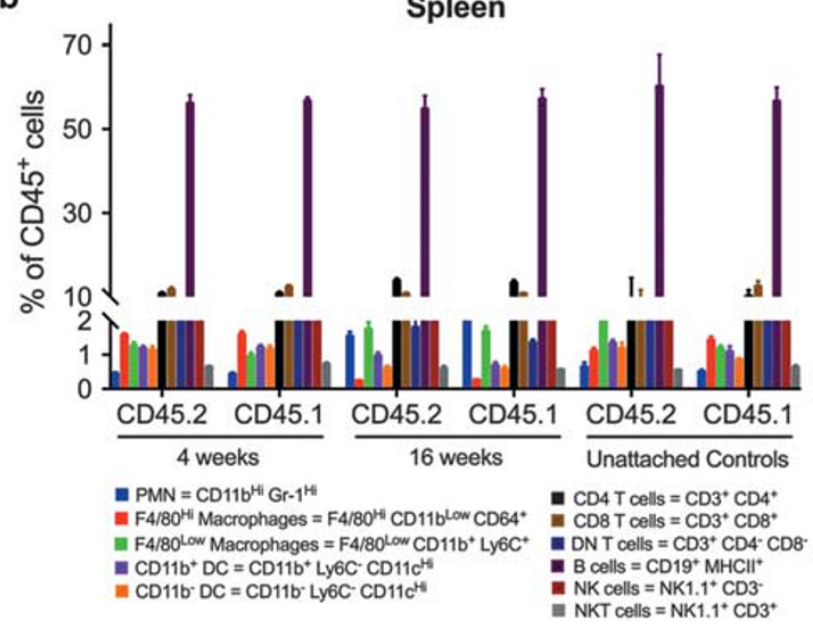

Figure 2 Distribution of leukocyte proportions in 4-week and 16-week CD45 congenic parabiotic chimeras and unattached controls, calculated as a percentage of viable $\mathrm{CD} 45^{+}$leukocytes. (a) Kidney and (b) spleen are shown. Abbreviated cell phenotypes with color coding are included. See detailed phenotypes in Table 1. Mean \pm s.e.m., $n=6$ pairs for CD45 congenic parabiotic chimeras, $n=2$ for each type of unattached control. Two-way ANOVA, Sidak's multiple comparisons test; ${ }^{*} P<0.05$ for proportion of given cell type after 16 weeks parabiosis vs same cell type after 4 weeks parabiosis. PMN, neutrophil; DC, dendritic cell; NK, natural killer.

and CD8s (Figure 2a). The proportion of B cells increased in the kidney of 16-week eGFP parabionts compared with 4week (Supplementary Figure 2a). B-cell proportions also increased in the spleen (Supplementary Figure 2b). Owing to changes observed in intrarenal immune phenotype in 16week parabionts, we concluded the 4-week time point was optimal for further studies.

\section{Leukocyte Chimerism is Equivalent in Parabionts}

Four weeks after attachment, there were no significant differences in percent chimerism for $\mathrm{CD}^{+} 5^{+}$cells between eGFP or CD45 congenic parabionts in blood, spleen, or kidney (Figures $3 \mathrm{a}$ and $\mathrm{b}$ ). Therefore, the exchange of blood leukocytes was symmetrical within a given pair. However, chimerism was more variable in eGFP parabionts (Figure 3a) compared with CD45 congenic pairs (Figure $3 \mathrm{~b}$ ), so we chose a

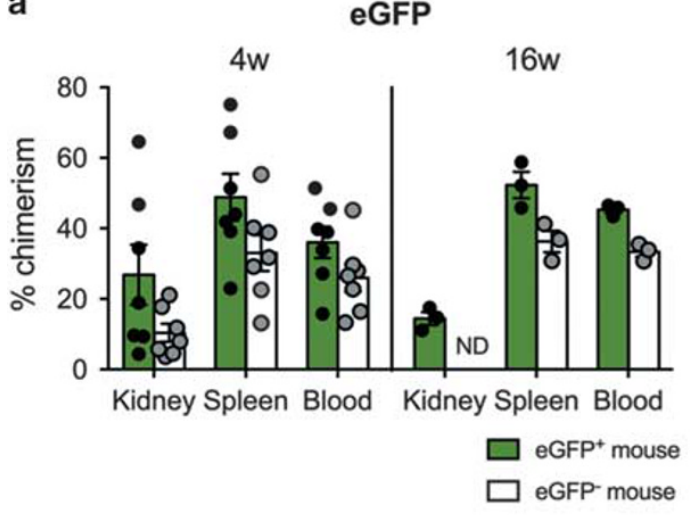

b

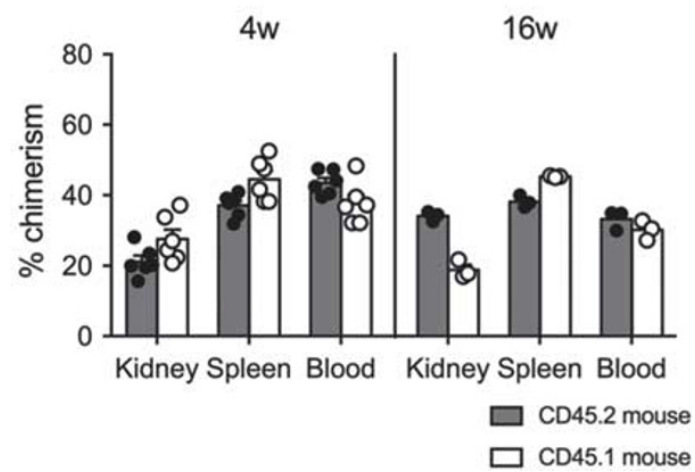

Figure $3 \mathrm{CD}^{+} 5^{+}$leukocyte percent chimerism in kidney, spleen, and blood after 4 weeks and 16 weeks of parabiosis. (a, b) Percent chimerism among $\mathrm{CD} 45^{+}$leukocytes in kidney, spleen, and blood from parabiotic chimeras; (a) eGFP system, (b) CD45 system. Each dot represents the mean of two measurements from an individual mouse. Data are from seven independent experiments. Mean \pm s.e.m., $n=7$ pairs for eGFP-4 weeks, $n=3$ pairs for eGFP- 16 weeks, $n=6$ pairs for CD45-4 weeks, $n=3$ pairs for CD45-16 weeks. ND, no data.

to utilize the CD45 congenic system primarily in further studies. We tested for the presence of anti-GFP antibodies in $\mathrm{eGFP}^{-/-}$parabionts using a flow cytometry-based assay, but they were not detectable above negative control levels (data not shown). One unexpected finding was lower percent chimerism in kidneys of CD45.1 mice compared with CD45.2 after 16 weeks of attachment $(18.8 \pm 1.5$ vs $34.0 \pm 0.8$, $P<0.05)$. Owing to low numbers of biologic replicates and a lack of differences among immune cell sub-populations, we believe this observation may have occurred as a result of a group effect. However, we cannot rule out that this statistical observation represents a true biologic process in the longterm parabionts. Despite the above-described difference, and of great importance to establishing valid time points for future studies, the variable of time (4 weeks $v s 16$ weeks) did not result in changes in percent chimerism in kidney, spleen, or blood (eg, CD45.2 mouse 4 weeks vs CD45.2 mouse 16 weeks; Figure $3 \mathrm{~b}$ ), validating that chimerism had reached a steady-state in these pairs after 4 weeks. 
a
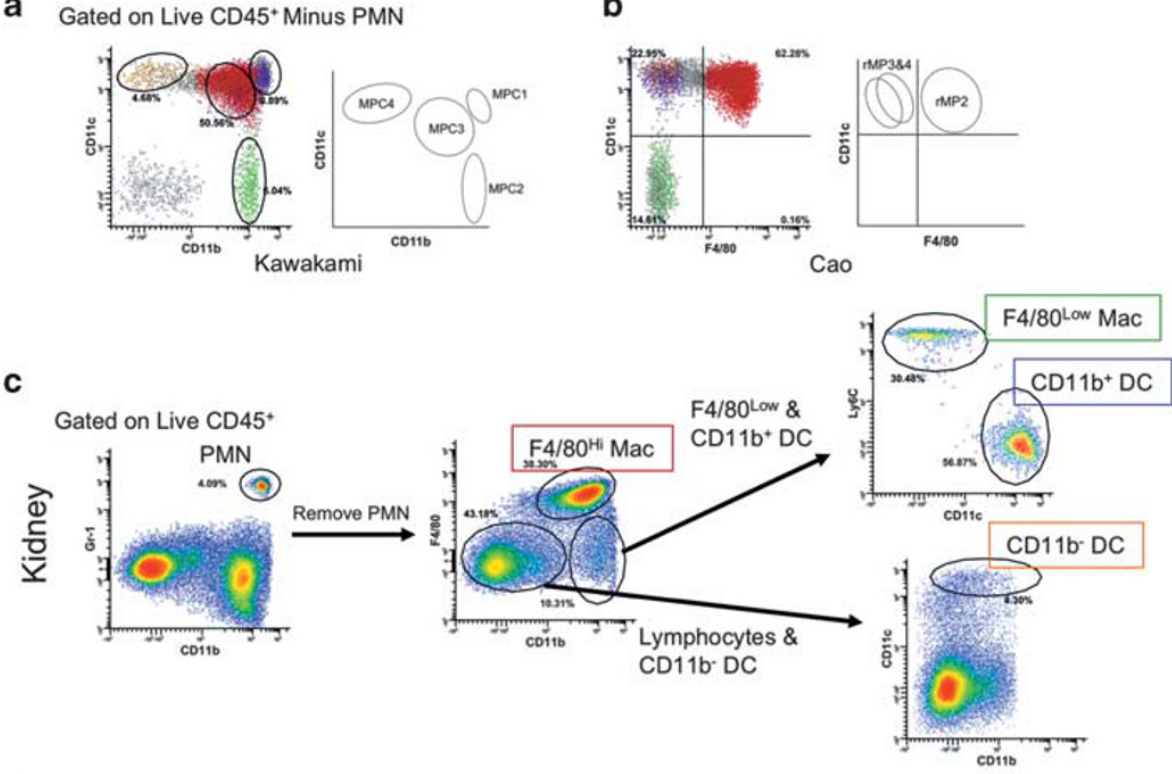

d

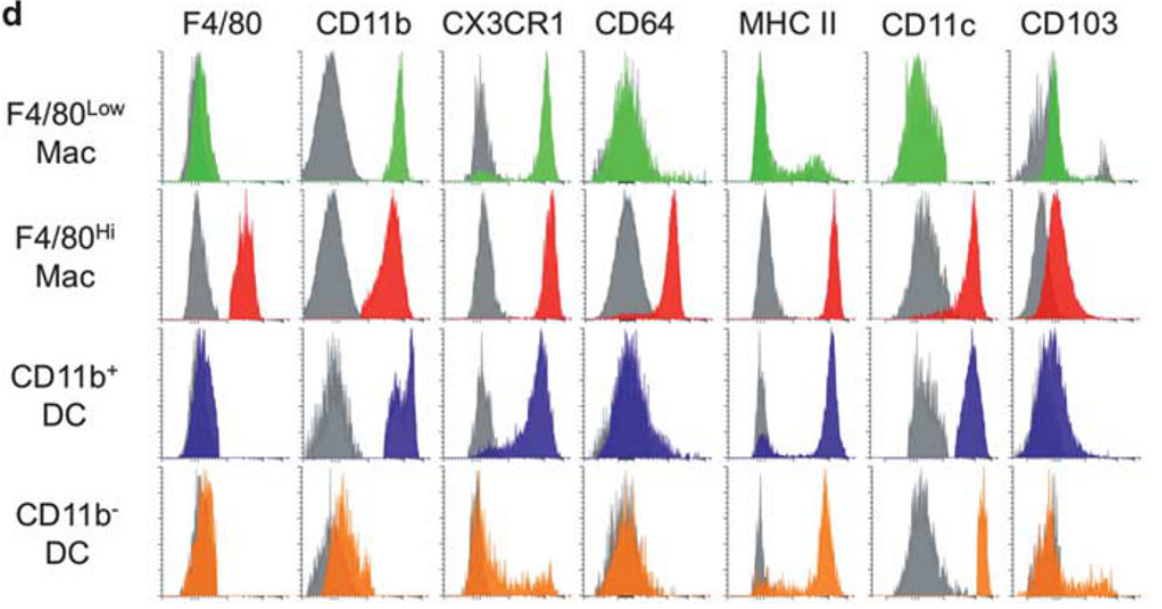

Figure 4 Method for identifying renal myeloid lineage inflammatory cells. Renal MP populations were identified based on previously published phenotypes. Flow histograms were plotted on (a) CD11b vs CD11c (see Kawakami et al ${ }^{30}$ ) and (b) F4/80 vs CD11c (see Cao et al ${ }^{15}$ ). (c) Flow cytometry gating strategy employed for identifying myeloid lineage cells, including PMN, F4/80 ${ }^{\mathrm{Hi}}$ macrophages, F4/80 ${ }^{\mathrm{Low}}$ macrophages, and DC in the kidney. Percent gated is shown for each region. (d) One parameter flow cytometry histograms demonstrate the surface phenotype of renal MP subsets. Gray histograms are mixed isotype and fluorescence minus one-stained controls from kidney single-cell suspensions for the given cell type. Histograms are representative of three independent experiments. PMN, neutrophil; Mac, macrophage; DC, dendritic cell.

\section{Myeloid Lineage Leukocytes in the Kidney were Subdivided into Host and Non-Host Chimeric Cells Based on Surface Phenotype}

Single-cell suspensions of renal leukocytes were prepared from mice after perfusion via left ventricular cardiac puncture with $60 \mathrm{ml}$ cold PBS. We used flow cytometry to determine the phenotype and quantify leukocytes in the kidney, applying a slightly modified gating strategy for identifying MP populations published by Kawakami and colleagues (Figure 4a). ${ }^{30}$ Further, the populations identified here demonstrate similar profiles compared with renal MP previously described by Cao et al (Figure 4b). ${ }^{31}$ Myeloid lineage cells consist of PMN, F4/80 ${ }^{\mathrm{Hi}}$ macrophages, F4/80 ${ }^{\text {Low }}$ macrophages (cells that are defined by the F4/80 ${ }^{\text {Low }} \mathrm{CD} 11 b^{\mathrm{Hi}} \mathrm{Ly}_{6 \mathrm{C}^{\mathrm{Hi}} \mathrm{CD} 11 \mathrm{c}^{-} \mathrm{MHCII}}{ }^{\mathrm{Low}} \mathrm{CD} 64^{-}$phenotype, which expand rapidly after AKI, and therefore are likely monocyte derived), and at least two subsets of DCs (Figure 4c). DCs were characterized by high expression of CD11c and MHC class II, lack of expression of CD64 (Fcylr), and were further subdivided based on their expression of the integrin, CD11b (Figure 4d, summarized in Table 1). ${ }^{30,31}$

\section{$\mathbf{F 4} / 80^{\mathrm{Hi}}$ Macrophages are Tissue Resident in the Kidney}

Myeloid cell sub-populations were assessed for expression of the CD45.1 or CD45.2 allotype, linking cells with their source 
Table 1 Kidney Leukocyte Surface Phenotypes

\begin{tabular}{|c|c|c|}
\hline Lineage & Cell type & Phenotype \\
\hline \multirow[t]{5}{*}{ Myeloid $^{\mathrm{a}}$} & PMN & $\mathrm{CD}_{4} 5^{+} \mathrm{CD} 11 \mathrm{~b}^{\mathrm{Hi}} \mathrm{Gr}_{-1}{ }^{\mathrm{Hi}}\left(\mathrm{F} 4 / 80^{-} \mathrm{MHCII}^{-}\right)$ \\
\hline & $\mathrm{F} 4 / 80^{\mathrm{Hi}}$ macrophages & $\mathrm{CD}_{4} 5^{+} \mathrm{F} 4 / 80^{\mathrm{Hi}} \mathrm{CD} 11 \mathrm{~b}^{\mathrm{Low}} \mathrm{Gr}^{-1^{-}}\left(\mathrm{MHCl} \mathrm{I}^{+} \mathrm{CD} 11 \mathrm{c}^{+} \mathrm{CX} 3 \mathrm{CR} 1^{+} \mathrm{CD} 4^{+}\right)$ \\
\hline & F4/80 Low macrophages & $\mathrm{CD}_{4} 5^{+} \mathrm{F} 4 / 80^{\mathrm{Low} /-} \mathrm{CD} 11 \mathrm{~b}^{+} \mathrm{Gr}-1^{-}$Ly6C $^{\mathrm{Hi}} \mathrm{CD} 11 \mathrm{C}^{-}\left(\mathrm{MHCII}^{\text {Low }} \mathrm{CX} \mathrm{CR} 1^{+} \mathrm{CD} 64^{-}\right)$ \\
\hline & $C D 11 b^{+} \mathrm{DC}$ & 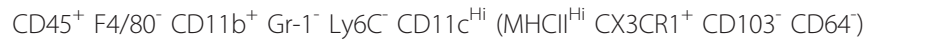 \\
\hline & $C D 11 b^{-} D C$ & 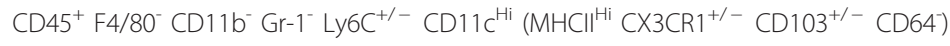 \\
\hline \multirow[t]{7}{*}{ Lymphoid } & B cells & $\mathrm{CD}_{4} 5^{+} \mathrm{CD} 19^{+} \mathrm{MHCl}^{+}$ \\
\hline & NK cells & $\mathrm{CD}_{4} 5^{+} \mathrm{CD} 19^{-} \mathrm{NK} 1.1^{+} \mathrm{CD}^{-}$ \\
\hline & NKT cells & $\mathrm{CD}_{4} 5^{+} \mathrm{CD} 19^{-} \mathrm{NK} 1.1^{+} \mathrm{CD}^{+}$ \\
\hline & T cells & $\mathrm{CD}^{+} 5^{+} \mathrm{CD}_{19}^{-} \mathrm{NK} 1.1^{-} \mathrm{MHCll}^{-} \mathrm{CD}^{+}$ \\
\hline & CD4 T cells & $\mathrm{CD}_{4} 5^{+} \mathrm{CD} 19^{-} \mathrm{NK} 1.1^{-} \mathrm{MHCll}^{-} \mathrm{CD}^{+} \mathrm{CD}^{+} \mathrm{CD} 8^{-}$ \\
\hline & CD8 T cells & $\mathrm{CD}_{4} 5^{+} \mathrm{CD} 19^{-} \mathrm{NK}_{1.1^{-}} \mathrm{MHCll}^{-} \mathrm{CD}^{+} \mathrm{CD}^{-} \mathrm{CD}^{+}$ \\
\hline & DNTLS & $\mathrm{CD}_{4} 5^{+} \mathrm{CD}_{19}^{-} \mathrm{NK} 1.1^{-} \mathrm{MHCll}^{-} \mathrm{CD}^{+} \mathrm{CD}^{-} \mathrm{CD}^{-}$ \\
\hline
\end{tabular}

Parentheses indicate surface markers not routinely used for sub-population quantification.

$+/-=$ The given population demonstrates both positive and negative sub-populations for the associated antigen.

Abbreviation: DNTLs, double-negative $T$ lymphocytes.

${ }^{\mathrm{a}} \mathrm{Gr}-1$ was detected using the $1 \mathrm{~A} 8$ monoclonal antibody clone.

animal. Percent chimerism was measured for the given populations (Figure 5a). Our ability to identify the source of intrarenal leukocytes was the basis for our measurement of percent chimerism, and therefore determining exchange with the blood for a given cell type.

Equivalent percent chimerism in kidney and spleen was interpreted as full exchange with the peripheral circulating immune cell pool. After 4 weeks of parabiosis, intrarenal PMN demonstrated full exchange with the spleen and blood (Figure $5 \mathrm{~b}$ and Supplementary Figure $3 \mathrm{a}$ ). The exchange behavior of kidney macrophages was notably different compared with PMN; macrophages demonstrated percent chimerism equal to $1.7 \pm 0.3 \%$ in $\mathrm{CD} 45$ parabionts (Figure 5c) and $1.5 \pm 0.9 \%$ in eGFP (Supplementary Figure $3 b$ ). Percent chimerism for kidney $\mathrm{F} 4 / 80^{\mathrm{Hi}}$ macrophages was significantly less than for splenic red pulp macrophages $\left(\mathrm{F} 4 / 80^{+} \mathrm{CD}^{+} 4^{+}\right.$) (Figure $5 \mathrm{c}$ ), kidney $\mathrm{F} 4 / 80^{\text {Low }}$ macrophages (Figure 5d), and kidney DC (Figure 5e). Decreased exchange for $\mathrm{F} 4 / 80^{\mathrm{Low}}$ macrophages was observed in eGFP parabionts after 4 weeks (Supplementary Figure 3c), and a similar trend was observed in CD45 parabionts (Figure 5d), indicating monocytes that patrol and/or transmigrate and differentiate in the kidney during quiescence may be long-lived. No exchange differences were observed between $\mathrm{Ly}_{6 \mathrm{C}} \mathrm{Hi}^{\mathrm{Hi}}$ and $\mathrm{Ly} 6 \mathrm{C}^{\mathrm{Int}} \mathrm{F} 4 / 80^{\mathrm{Low}}$ macrophages in quiescent kidneys, so they were grouped together in this analysis. PMN and $\mathrm{F} 4 / 80^{\mathrm{Low}}$ macrophages demonstrated decreased exchange in 16-week parabionts compared with 4-week (Figures $5 \mathrm{~b}$ and d), which suggests 4-16 weeks may represent the useful window within which the parabiosis model can be applied for the study of myeloid cell-mediated renal inflammation. It is possible that a size barrier in anastomotic vessels contributed to the decreased chimerism observed for PMN and F4/80 ${ }^{\mathrm{Low}}$ macrophages in the 16-week parabionts. Although a previous study by Gibney et al purports a size barrier does not exist, their study was limited to 1 month of parabiotic attachment. ${ }^{32}$

\section{Intrarenal CD11b- DCs Demonstrate Lower Percent Chimerism than $\mathrm{CD}_{11 \mathrm{~b}^{+} \mathrm{DCs}}$}

After 4 weeks of parabiosis, in CD45 congenic parabionts, intrarenal $\mathrm{CD}_{11} \mathrm{~b}^{+} \mathrm{DC}$ demonstrate greater exchange than $\mathrm{CD} 1 \mathrm{~b}^{-} \mathrm{DC}$ (Figure 5e) and a similar trend was observed in eGFP parabionts (Supplementary Figure 3d). However, DC subtypes demonstrated equivalent percent chimerism in the kidney and spleen, indicating DC exchange is comparable in the kidney and secondary lymphoid tissue, where antigen presentation to lymphocytes is commonly performed. Kawakami et al demonstrated that both $\mathrm{CD}_{11} \mathrm{~b}^{-}$and $\mathrm{CD}_{11 b^{+}}$DC emigrate from the kidney in response to intraperitoneal LPS administration. ${ }^{30}$ Cao et al demonstrated that $\mathrm{CD} 11 \mathrm{~b}^{-} \mathrm{CD} 103^{+} \mathrm{DC}$ were primarily responsible for $\mathrm{CD} 8$ T-cell activation in response to adriamycin nephropathy. ${ }^{15}$ Thus, both DC subtypes have the potential to migrate to secondary lymphoid organs, but the difference in kidney

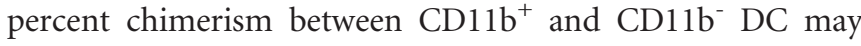
represent an additional functional difference between these cell types. 
a
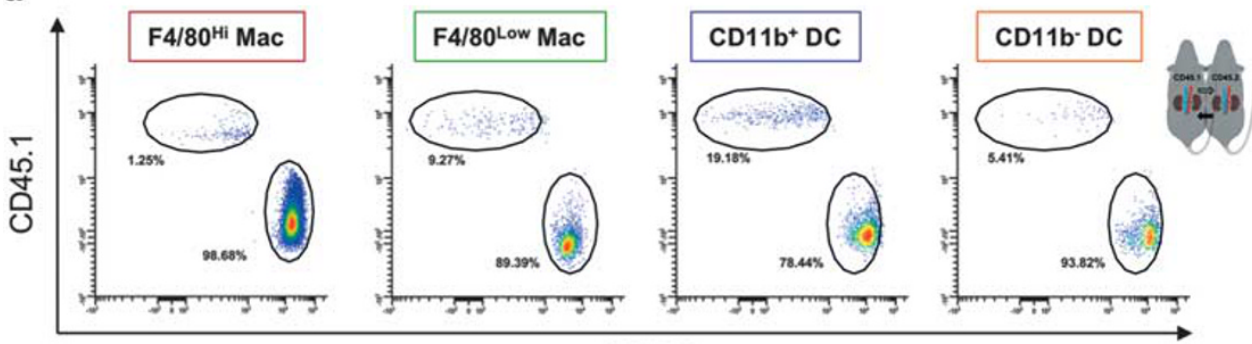

CD45.2
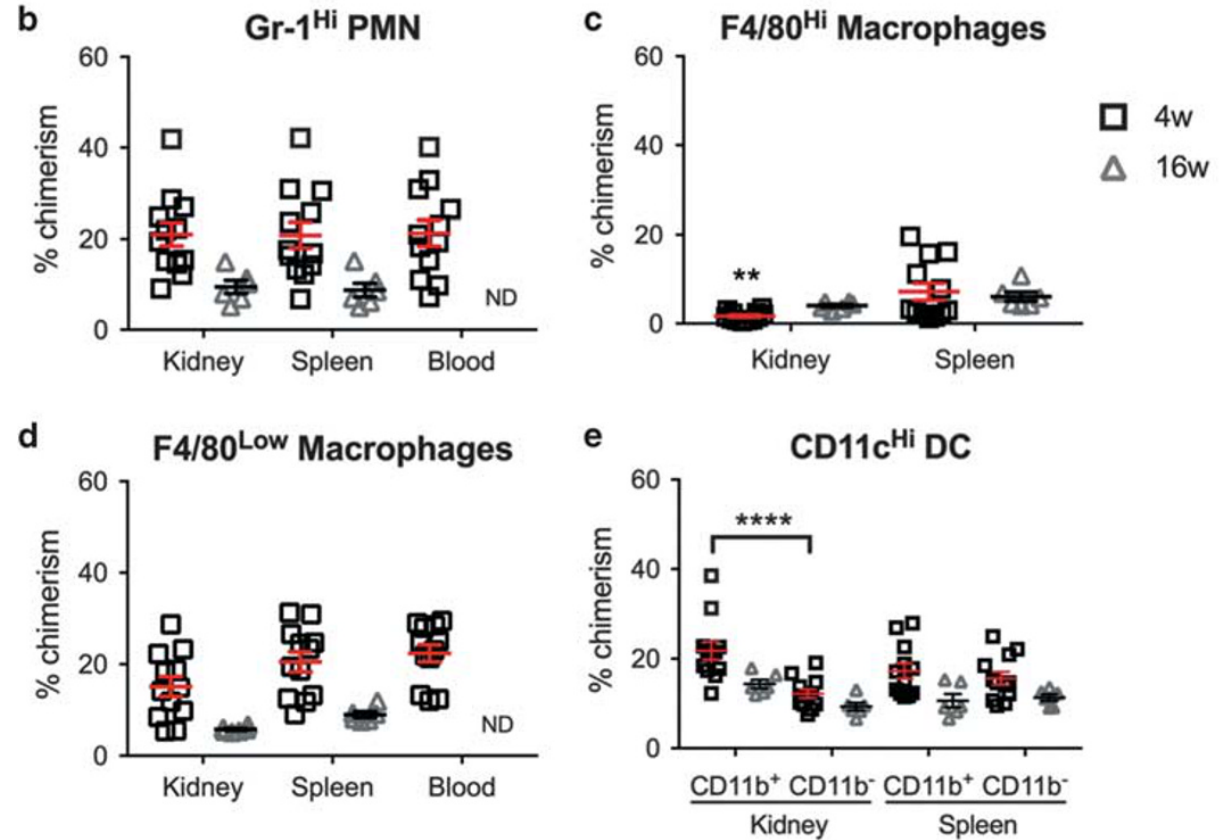

Figure 5 Percent chimerism for myeloid lineage cells from kidney, spleen, and blood of CD45 parabionts. (a) Representative flow cytometry histograms of chimeric populations of renal MPs. Quantification of percent chimerism for (b) PMN, (c) F4/80 ${ }^{\mathrm{Hi}}$ macrophages, (d) F4/80 $0^{\mathrm{Low}}$ macrophages, and (e)

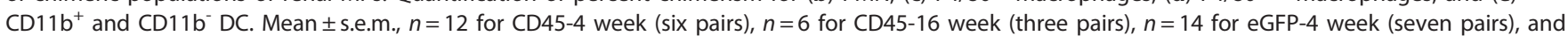
$n=3$ for eGFP-16 week (three pairs). Two-way ANOVA, Sidak's multiple comparisons test, comparing kidney vs spleen for each cell type at each time point, ${ }^{* *} P<0.01,{ }^{* * *} P<0.0001$. PMN, neutrophil; Mac, macrophage; DC, dendritic cell; ND, no data.

\section{A Fraction of T Lymphocytes and NKT Cells are Tissue Resident or Long-Lived in the Kidney}

Lymphocytes were quantified from the same samples prepared in parallel from the same kidneys as those used for myeloid lineage cell analysis. Host and non-host, chimeric B lymphocytes, NK cells, NKT cells, and T lymphocytes (subdivided into $\mathrm{CD}^{+}, \mathrm{CD}^{+}$, and $\mathrm{CD} 4^{-} \mathrm{CD}^{-}$double negative, DNTL) were identified (Figures $6 \mathrm{a}$ and b,Table 1).

The differentiation and retention of functionally unique tissue-resident lymphocytes in non-lymphoid tissues have been described in gut, skin, liver, lung, and kidney, and these cells have unique function compared with their circulating counterparts. ${ }^{9,33,34}$ After 4 weeks of parabiosis in CD45 parabionts, $\mathrm{CD}^{+} \mathrm{T}$ cells demonstrated significantly lower percent chimerism in kidney compared with spleen and blood (Figure $6 \mathrm{c}$ ). Among $\mathrm{CD}^{+}{ }^{\mathrm{T}}$ cells, percent chimerism of $\mathrm{CD}^{+}, \mathrm{CD}^{+}$, and DNTL were quantified (Figures 6d-f). In
CD45 parabionts, CD4 ${ }^{+} \mathrm{T}$ cells and DNTLs demonstrated lower percent chimerism in kidney than in spleen after 4 weeks of parabiosis (Figures $6 \mathrm{~d}$ and $\mathrm{f}$ ). In long term, 16week parabionts, CD4 percent chimerism in the kidney increased to reach equivalence with the spleen (Figure $6 \mathrm{~d}$ ), indicating the difference at 4 weeks may have been due to long-lived, memory $\mathrm{T}$ cells, rather than tissue-resident cells. After 16 weeks of parabiosis, the percent chimerism of DNTL in the kidney was equivalent with that of the spleen, however, there was a statistical trend toward DNTL percent chimerism remaining significantly lower in kidney than in spleen (Figure 6f). In eGFP parabionts, the percent chimerism of DNTL after 16 weeks of parabiosis remained significantly decreased relative to spleen (Supplementary Figure 4d). However, percent chimerism observed for intrarenal lymphocytes in eGFP parabionts demonstrated greater variability for most cell types (Supplementary Figure 4). NKT cells 
a

Gated on Live CD45+
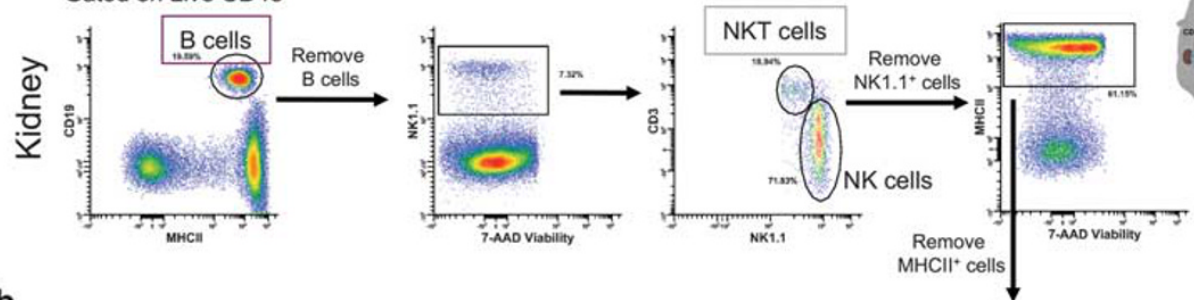

b
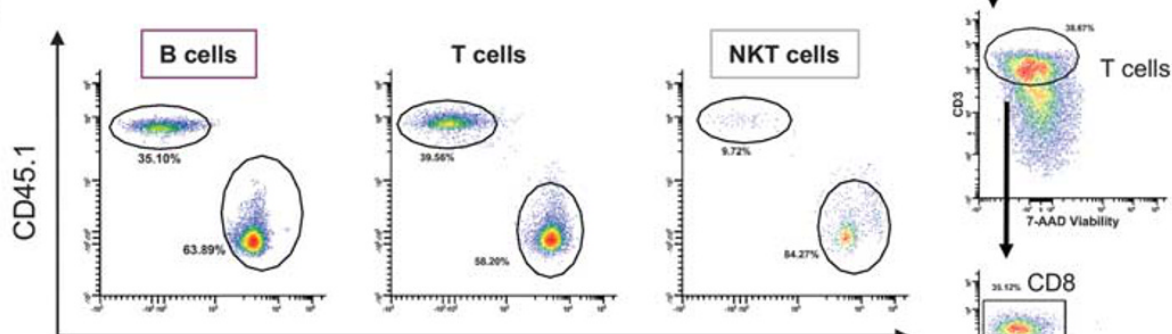

CD45.2
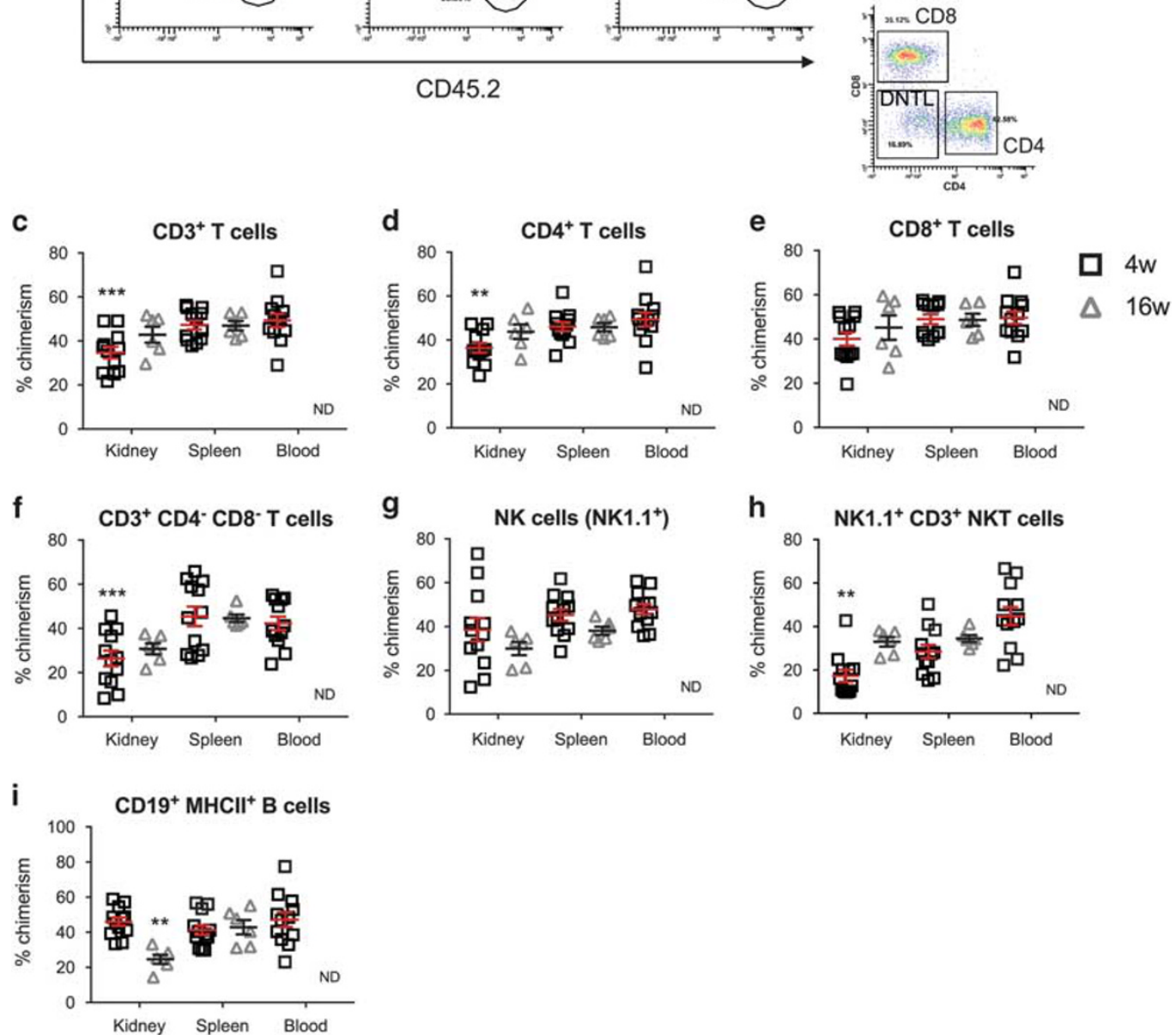

Figure 6 (a) Gating strategy for kidney lymphoid lineage cells from quiescent CD45 parabionts, including B, NK, NKT, T, CD4 and CD8 T cells, and DNTL. Percent gated is shown for each region. (b) Representative flow cytometry histograms of chimeric populations of lymphocytes observed in the kidney. (c-i) Quantification of percent chimerism for (c) T cells, (d) CD4, (e) CD8, (f) DNTLs, (g) NK cells, (h) NKT cells, and (i) B cells from kidney, spleen, and blood of CD45 parabionts. Mean \pm s.e.m., $n=12$ for $4 \mathrm{w}$ and $n=6$ for $16 \mathrm{w}$. Two-way ANOVA, Sidak's test for multiple comparisons, comparing kidney vs spleen for each cell type at each time point, ${ }^{* *} P<0.01,{ }^{* *} P<0.001$. ND, no data; NK, natural killer; DNTL, double-negative T lymphocyte.

demonstrated lower percent chimerism in kidney than in blood but not spleen (Figure 6h). Interestingly, NKT cells demonstrated relatively low percent chimerism in spleen of both CD45 and eGFP parabionts at the 4-week time point (Figure 6h and Supplementary Figure 4f).

\section{B-Cell Chimerism Decreases in the Kidney in Long-Term Parabiosis Experiments}

B-cell infiltration can impede repair after $\mathrm{AKI}^{35}$ and is present in intrarenal tertiary lymphoid structures in the context of lupus nephritis, as well as in AKI in aged mice. ${ }^{12,36}$ We found 
that intrarenal B-cell proportions increased in long-term parabiosis pairs, in both the CD45 and eGFP systems (Figure 2a and Supplementary Figure 2a). At the 4-week time point, B lymphocytes demonstrated full exchange with the blood, as indicated by equivalent percent chimerism in kidney, spleen, and blood (Figure 6i and Supplementary Figure $4 \mathrm{~g}$ ). However, at the 16-week time point, percent chimerism for B cells was significantly reduced in kidney when compared with spleen (Figure 6i and Supplementary Figure $4 \mathrm{~g}$ ). This finding, in combination with changes in intrarenal proportions, indicates accumulation of hostderived B lymphocytes in the kidney after 16 weeks of parabiosis, further supporting that parabiosis has a useful window within which it can be applied for the study of renal inflammation.

\section{DISCUSSION}

Many pathologies that afflict the kidney are driven or facilitated by inflammation and immune-mediated effects. Thus, leukocyte dynamics in the kidney are of great interest. The primary intent of this study was to answer the fundamental question about the extent to which renal leukocytes exchange with the systemic immune cell pool. To answer this question and to better understand leukocyte dynamics in the kidney, we employed the parabiosis model, which allows specific and permanent labeling of leukocytes coming from the peripheral blood. We found that CD45 congenic mice (Figure $3 \mathrm{~b}$ ) demonstrated less variability in percent chimerism than eGFP transgenic parabionts (Figure 3a).

For most immune cell types in the quiescent kidney, comparable percent chimerism was measured in spleen and kidney, which is significant, because the spleen is a secondary lymphoid organ through which immune cells traffic, as opposed to the kidney, which is a non-lymphoid organ. A cell type that demonstrates equivalent chimerism in kidney and spleen is likely derived from bone marrow precursors or mature circulating cells. It appears neutrophils, monocytederived cells, certain types of DCs, B cells, CD4 and CD8 $\mathrm{T}$ cells, and NK cells all perform immune surveillance within the dense parenchyma of the healthy kidney. Putatively, the kidney does not have a large role in development of the systemic immune system or maintenance of self-antigen tolerance, however, the extent, diversity, and dynamic exchange of the renal immune cell pool had not yet been described before this study. The role of resident immune cells in maintaining tolerance to renal self-antigens may be underappreciated.

We found that several immune cell types from both the myeloid and lymphoid lineages demonstrate full exchange with the systemic immune cell pool. However, F $4 / 80^{\mathrm{Hi}}$ macrophages, are minimally exchanged with circulating cells during quiescence (Figure $5 c$ and Supplementary Figure $3 b$ ). Tissue-resident and infiltrating macrophages have somewhat opposing roles in the context of AKI. The transition of kidney macrophages from damage-promoting to pro-resolving cells is vital for recovery from acute injury. ${ }^{3,16,18}$ Damagepromoting macrophages are characterized by expression of iNOS, response to IFN-gamma, and associated with Th1/ Th17 cell activity. ${ }^{37-39}$ Pro-resolving macrophages are characterized by production of arginase- 1 and mannose receptor, response to granulocyte-macrophage colony-stimulating factor, are associated with IL-10 expression and resolution of inflammatory infiltrate, tissue regeneration, and restoration of kidney function. ${ }^{14,18,37,38}$ However, conclusions regarding the source of macrophage sub-populations in the kidney have been controversial and mostly dependent on surface markers and comparing numbers of macrophages during quiescence and at time points after injury. From this study, it is clear that the presence of immune cells in the kidney during quiescence does not establish tissue residence (as defined by semipermanent presence in the tissue and renewal independent of the bone marrow). In many other organ systems, such as the liver or the skin, tissue-resident macrophages proliferate in situ during normal physiology independent of hematopoietic precursors. It is likely that this is also true for kidney macrophages. However, the extent to which kidney $\mathrm{F} 4 / 80^{\mathrm{Hi}}$ macrophages proliferate in situ, as opposed to being supplemented by bone marrow-derived cells, in the setting of acute or chronic inflammation is not known. Near total expression of CX3CR1 by $\mathrm{F} 4 / 80^{\text {Low }}$ macrophages and

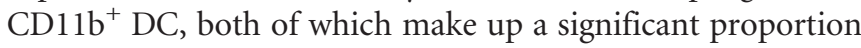
of the renal MP population and exchange freely, demonstrate serious hindrances to interpreting dynamics of kidneyresident macrophages in the CX3CR1 reporter mouse (Figure $4 \mathrm{~d}){ }^{8}$

Intrarenal lymphocytes demonstrated near full exchange with the blood, with some exceptions (Figure 6). Tissueresident effector-memory $\mathrm{T}$ cells have been described in nonlymphoid tissues, ${ }^{33}$ and $\mathrm{T}$ regulatory cells are important for coordinating suppression of the immune response to AKI and can be protective in this setting. ${ }^{40,41}$ Owing to the absence of alloantigen in IRI models, questions regarding the function of antigen-specific lymphocytes observed in the kidney after injury have arisen. ${ }^{42,43}$ DNTL have garnered interest as resident renal leukocytes, which are activated $\left(\mathrm{CD} 9^{+}\right)$during quiescence and are able to inhibit CD4 T-cell proliferation. ${ }^{24}$ NK cells are dynamically regulated in the acute response to renal IRI $^{44}$ and $\mathrm{NK}$ cell depletion protects from kidney dysfunction secondary to IRI. ${ }^{23}$

In the future, we plan to examine localization and mechanisms of leukocyte transmigration into and out of the kidney. The extent and behavior of trafficking of various subpopulations within the renal tissue remain to be determined. With respect to adaptive immune cells that surveil the kidney, the antigen specificity determined by $\mathrm{T}$ - or B-cell receptor repertoire, and receptors that may be responsible for homing cells to kidney tissue are unknown and represent intriguing areas for investigation. Studies of T-cell homing to the gut during colitis have identified G-protein-coupled receptor 15 
as important for targeting to the appropriate tissue. ${ }^{45}$ Leukocyte egress from the kidney has also not been thoroughly described. Additional pending studies will focus on leukocyte dynamics in the acute, subacute, and chronic inflammatory phases in recovery from AKI.

Parabiosis allows the study of renal leukocyte exchange with the blood, and helps elucidate fundamental cellular trafficking behavior in healthy kidneys. F4/80 $\mathrm{Hi}$ macrophages make up the largest proportion of kidney leukocytes during quiescence and they are not replaced with blood-derived precursors, whereas other cell types generally exchange freely. The study of renal leukocyte dynamics promises to expand our potential for targeting specific inflammatory cell populations in the treatment of kidney disease.

Supplementary Information accompanies the paper on the Laboratory Investigation website (http://www.laboratoryinvestigation.org)

\section{ACKNOWLEDGMENTS}

We acknowledge the UAB-UCSD O'Brien Center for acute kidney injury research (P30 DK079337), including Dr Gary Cutter for statistical analysis consultation; Drs Vidya Sagar Hanumantu and John Mountz and the UAB Comprehensive Flow Cytometry Core (P30 AR048311 and P30 A127667); and grant support from NIH R01 DK59600 (to AA and JFG), 16GRNT31180023 (to JFG), NIGMS MSTP T32GM008361 (to Dr Robin Lorenz for JML), and AHA 17PRE33370121 (to JML). We acknowledge somersault18:24 for the mouse graphic. We also acknowledge Dr Travis D Hull for intellectual contributions.

\section{DISCLOSURE/CONFLICT OF INTEREST}

The authors declare no conflict of interest.

1. Ernandez T, Mayadas TN. The changing landscape of renal inflammation. Trends Mol Med 2016;22:151-163.

2. Kurts $\mathrm{C}$, Panzer $\mathrm{U}$, Anders $\mathrm{HJ}$, et al. The immune system and kidney disease: basic concepts and clinical implications. Nat Rev Immunol 2013:13:738-753.

3. Huen SC, Cantley LG. Macrophages in renal injury and repair. Annu Rev Physiol 2017;79:449-469.

4. Kopp JB. Rethinking hypertensive kidney disease: arterionephrosclerosis as a genetic, metabolic, and inflammatory disorder. Curr Opin Nephrol Hypertens 2013;22:266-272.

5. Wada J, Makino H. Inflammation and the pathogenesis of diabetic nephropathy. Clin Sci (Lond) 2013;124:139-152.

6. Zhang MZ, Yao B, Yang S, et al. Csf-1 signaling mediates recovery from acute kidney injury. J Clin Invest 2012;122:4519-4532.

7. Soos TJ, Sims TN, Barisoni L, et al. Cx3cr1+ interstitial dendritic cells form a contiguous network throughout the entire kidney. Kidney Int 2006;70:591-596.

8. Stamatiades EG, Tremblay ME, Bohm M, et al. Immune monitoring of trans-endothelial transport by kidney-resident macrophages. Cell 2016;166:991-1003.

9. Mackay LK, Minnich M, Kragten NA, et al. Hobit and blimp1 instruct a universal transcriptional program of tissue residency in lymphocytes. Science 2016;352:459-463.

10. George J, Lever JM, Agarwal A. Mononuclear phagocyte subpopulations in the mouse kidney. Am J Physiol Renal Physiol 2017;312:F640-F646.

11. Leaf IA, Nakagawa $S$, Johnson BG, et al. Pericyte myd88 and irak4 control inflammatory and fibrotic responses to tissue injury. J Clin Invest 2017:127:321-334.

12. Sato $Y$, Mii A, Hamazaki $Y$, et al. Heterogeneous fibroblasts underlie age-dependent tertiary lymphoid tissues in the kidney. JCI Insight 2016;1:e87680.
13. Inoue $\mathrm{T}$, Abe $\mathrm{C}$, Sung SS, et al. Vagus nerve stimulation mediates protection from kidney ischemia-reperfusion injury through alpha7nachr+ splenocytes. J Clin Invest 2016;126:1939-1952.

14. Li L, Huang L, Sung SS, et al. The chemokine receptors CCR2 and CX3CR1 mediate monocyte/macrophage trafficking in kidney ischemia-reperfusion injury. Kidney Int 2008;74:1526-1537.

15. Cao $Q$, Wang $Y$, Wang $X M$, et al. Renal f4/80+ CD11c+ mononuclear phagocytes display phenotypic and functional characteristics of macrophages in health and in adriamycin nephropathy. J Am Soc Nephrol 2015:26:349-363.

16. Hull TD, Kamal Al, Boddu R, et al. Heme oxygenase-1 regulates myeloid cell trafficking in AKI. J Am Soc Nephrol 2015;26:2139-2151.

17. Ferenbach DA, Sheldrake TA, Dhaliwal K, et al. Macrophage/monocyte depletion by clodronate, but not diphtheria toxin, improves renal ischemia/reperfusion injury in mice. Kidney Int 2012;82:928-933.

18. Huen SC, Huynh L, Marlier A, et al. GM-CSF promotes macrophage alternative activation after renal ischemia/reperfusion injury. J Am Soc Nephrol 2015;26:1334-1345.

19. Schulz C, Gomez Perdiguero E, Chorro L, et al. A lineage of myeloid cells independent of myb and hematopoietic stem cells. Science 2012;336:86-90.

20. Gomez Perdiguero E, Klapproth K, Schulz C, et al. Tissue-resident macrophages originate from yolk-sac-derived erythro-myeloid progenitors. Nature 2015;518:547-551.

21. Mass E, Ballesteros I, Farlik M, et al. Specification of tissue-resident macrophages during organogenesis. Science 2016;353:aaf4238.

22. Hashimoto D, Chow A, Noizat $C$, et al. Tissue-resident macrophages self-maintain locally throughout adult life with minimal contribution from circulating monocytes. Immunity 2013:38:792-804.

23. Victorino F, Sojka DK, Brodsky KS, et al. Tissue-resident NK cells mediate ischemic kidney injury and are not depleted by anti-asialogm1 antibody. J Immunol 2015;195:4973-4985.

24. Martina MN, Noel S, Saxena A, et al. Double-negative alphabeta T cells are early responders to aki and are found in human kidney. J Am Soc Nephrol 2016;27:1113-1123.

25. Bunster E, Meyer RK. An improved method of parabiosis. The Anatomical Record 1933;57:339-343.

26. Kamran $\mathrm{P}$, Sereti $\mathrm{Kl}$, Zhao $\mathrm{P}$, et al. Parabiosis in mice: a detailed protocol. J Vis Exp 2013;80:e50556.

27. Hull TD, Bolisetty S, DeAlmeida AC, et al. Heme oxygenase-1 expression protects the heart from acute injury caused by inducible cre recombinase. Lab Invest 2013:93:868-879.

28. Takahashi N, Boysen G, Li F, et al. Tandem mass spectrometry measurements of creatinine in mouse plasma and urine for determining glomerular filtration rate. Kidney Int 2007;71:266-271.

29. Schaefer BC, Schaefer ML, Kappler JW, et al. Observation of antigendependent CD8+ T-cell/dendritic cell interactions in vivo. Cell Immunol 2001;214:110-122.

30. Kawakami T, Lichtnekert J, Thompson $\mathrm{U}$, et al. Resident renal mononuclear phagocytes comprise five discrete populations with distinct phenotypes and functions. J Immunol 2013;191:3358-3372.

31. Cao Q, Lu J, Li Q, et al. Cd103+ dendritic cells elicit CD8+ T cell responses to accelerate kidney injury in adriamycin nephropathy. J Am Soc Nephrol 2016;27:1344-1360.

32. Gibney BC, Chamoto K, Lee GS, et al. Cross-circulation and cell distribution kinetics in parabiotic mice. J Cell Physiol 2012;227:821-828.

33. Casey KA, Fraser KA, Schenkel JM, et al. Antigen-independent differentiation and maintenance of effector-like resident memory T cells in tissues. J Immunol 2012;188:4866-4875.

34. Wu T, Hu Y, Lee YT, et al. Lung-resident memory Cd8 Tcells (TRM) are indispensable for optimal cross-protection against pulmonary virus infection. J Leukoc Biol 2014:95:215-224.

35. Jang HR, Gandolfo MT, Ko GJ, et al. B cells limit repair after ischemic acute kidney injury. J Am Soc Nephrol 2010;21:654-665.

36. Kang S, Fedoriw $\mathrm{Y}$, Brenneman EK, et al. Baff induces tertiary lymphoid structures and positions $T$ cells within the glomeruli during lupus nephritis. J Immunol 2017;198:2602-2611.

37. Lee $\mathrm{S}$, Huen $\mathrm{S}$, Nishio $\mathrm{H}$, et al. Distinct macrophage phenotypes contribute to kidney injury and repair. J Am Soc Nephrol 2011;22:317-326.

38. Wynn TA, Vannella KM. Macrophages in tissue repair, regeneration, and fibrosis. Immunity 2016:44:450-462.

39. Ferenbach DA, Bonventre JV. Mechanisms of maladaptive repair after AKI leading to accelerated kidney ageing and ckd. Nat Rev Nephrol 2015;11:264-276. 
40. Gandolfo MT, Jang HR, Bagnasco SM, et al. Foxp3+ regulatory T cells participate in repair of ischemic acute kidney injury. Kidney Int 2009;76:717-729.

41. Kinsey GR, Sharma R, Huang L, et al. Regulatory $T$ cells suppress innate immunity in kidney ischemia-reperfusion injury. J Am Soc Nephrol 2009;20:1744-1753.

42. Ascon DB, Ascon M, Satpute $S$, et al. Normal mouse kidneys contain activated and $\mathrm{cd} 3+\mathrm{cd} 4-\mathrm{cd} 8$ - double-negative $\mathrm{t}$ lymphocytes with a distinct TCR repertoire. J Leukoc Biol 2008;84:1400-1409.
43. Satpute SR, Park JM, Jang HR, et al. The role for T cell repertoire/ antigen-specific interactions in experimental kidney ischemia reperfusion injury. J Immunol 2009;183:984-992.

44. Ascon DB, Lopez-Briones S, Liu M, et al. Phenotypic and functional characterization of kidney-infiltrating lymphocytes in renal ischemia reperfusion injury. J Immunol 2006;177:3380-3387.

45. Nguyen LP, Pan J, Dinh TT, et al. Role and species-specific expression of colon T cell homing receptor gpr15 in colitis. Nat Immunol 2015;16: 207-213. 